\title{
Carqueja (Baccharis trimera): utilização terapêutica e biossíntese
}

\author{
KARAM, T.K. ${ }^{1}$; DALPOSSO, L.M. ${ }^{1}$; CASA, D.M. ${ }^{1}$; DE FREITAS, G.B.L. ${ }^{12^{\star}}$ \\ ${ }^{1}$ Departamento de Farmácia, Universidade Estadual do Centro-Oeste, Rua Simeão Camargo Varela de Sá, 03 \\ , Vila Carli, CEP: 85045-000, Guarapuava - Paraná 'Instituto de Química, LABMMOL, Universidade Federal do \\ Rio de Janeiro, Avenida Athos da Silveira Ramos, 149 Bloco A - $7^{\circ}$ andar. Cidade Universitária, CEP: 21941- \\ 909, Rio de Janeiro - Rio de Janeiro. "guilhermebarroso@pop.com.br
}

\begin{abstract}
RESUMO: A utilização de plantas medicinais no tratamento e cura de enfermidades é um recurso terapêutico muito antigo e talvez o único adotado em muitas comunidades e grupos étnicos. A família Asteraceae concentra grande número de espécies com potencial terapêutico, algumas das quais do gênero Baccharis, utilizadas na medicina popular e também na produção de fitoterápicos. Estudos relatam que a principal indicação terapêutica mencionada para estas espécies estão relacionadas com ações sobre o trato gastrintestinal. A partir de pesquisa bibliográfica, foram identificadas as características morfológicas da carqueja, bem como, as propriedades terapêuticas cientificamente comprovadas, biossíntese dos principais metabólitos secundários, e possíveis interações medicamentosas.
\end{abstract}

Palavras-chave: planta medicinal, morfologia, fitoterápico, metabólito

\begin{abstract}
Broom (Baccharis trimera): therapeutic use and biosynthesis. The use of medicinal plants to treat and cure diseases is a very old therapeutic resource and perhaps the only one adopted in many communities and ethnic groups. The Asteraceae family concentrates a great number of species with therapeutic potential, some of which belong to the Baccharis genus and have been used in folk medicine and herbal medicine production. Studies have reported that the main therapeutic indication for these species is related to actions on the gastrointestinal tract. From literature searches, the morphological characteristics of broom, its scientifically proven therapeutic properties, as well as biosynthesis of secondary metabolites and possible drug interactions were identified.
\end{abstract}

Keywords: medicinal plant, morphology, phytotherapic, metabolite

\section{INTRODUÇÃO}

O homem sempre buscou na natureza soluções para os problemas de saúde. A utilização das plantas medicinais na prevenção e tratamento das doenças é feita desde os tempos mais remotos da humanidade. De fato, descobertas realizadas junto aos restos mortais dos primeiros hominídeos comprovam que há cerca de 60.000 anos atrás já se utilizavam, para fins medicinais, diversas plantas.

Durante milênios, o recurso à fitoterapia, fármacos de origem vegetal, correspondeu a cerca de $90 \%$ dos medicamentos utilizados para o alívio e cura das doenças (Cunha et al., 2003). Ao considerar o medicamento sob o ponto de vista cronológico, constata-se a preponderância na utilização das plantas até cerca de 1930, época de desenvolvimento das primeiras sulfamidas, ponto de partida para o surgimento da geração da síntese orgânica de fármacos com grande número de moléculas sintéticas (Marques, 2008).

Somente no final do século XVIII, inicia o isolamento e a determinação da estrutura dos constituintes ativos dos produtos de origem natural dotados de propriedades medicinais. Embora, já tivessem sido isolados o ácido benzóico, a sacarose, a cânfora e o timol, foram os trabalhos realizados por volta de 1770 pelo pesquisador sueco Scheele, que deram início a esta nova etapa com a obtenção de vários ácidos orgânicos e também a lactose e a glicerina, todos obtidos a partir de produtos naturais. Com o isolamento dos constituintes dotados de ação farmacológica, entra-se numa nova fase da utilização científica das plantas medicinais, com a substituição progressiva destas e dos extratos, pelos compostos reconhecidos como responsáveis pela ação farmacológica (Cunha, 2005).

Plantas medicinais, assim como os

Recebido para publicação em 17/05/2011

Aceito para publicação em 15/12/2011

Rev. Bras. PI. Med., Campinas, v.15, n.2, p.280-286, 2013. 
medicamentos sintéticos, possuem grupos de compostos farmacologicamente ativos que atuam nos organismos vivos. O emprego terapêutico dessas plantas exige o conhecimento prévio dos compostos para a avaliação das potencialidades terapêuticas. A toxicidade também é de grande importância, para conseguir a formulação apropriada, assim como a estratégia adequada para o uso sem maiores riscos. As plantas, utilizadas popularmente devido às atividades medicinais, podem conter substâncias que oferecem ação tóxica, mutagênica e até carcinogênica se utilizadas de maneira indiscriminada. Equivocadamente a cultura popular acredita que fazer uso exagerado de determinada planta não causa mal algum, pelo simples fato desta ser de origem natural (Peron et al., 2008).

No Brasil as plantas medicinais são consideradas "remédios de baixo custo", o que no país é encarado como sinônimo de baixa qualidade. Grande parte das espécies vegetais utilizadas pela população brasileira não possui ação farmacológica comprovada, estudo químico realizado e nem mesmo estudos toxicológicos (Bacchi,1996). O mercado brasileiro ainda se encontra seriamente restrito devido à falta de pesquisa e desenvolvimento, bem como de investimentos na área de controle de qualidade de produtos padronizados (Vilegas \& Cardoso, 2007). Entretanto, estudos comprovam atividade terapêutica de-diversas espécies medicinais.

Parte da população brasileira já se habituou à utilização de espécies vegetais amargas para problemas hepáticos ou relacionados à digestão, sendo assim, a Baccharis trimera, popularmente conhecida como carqueja, é uma das espécies mais utilizadas nesta ocasião (Borella et al., 2006). O primeiro registro escrito do uso no Brasil data de 1931, informando o emprego da infusão das folhas e ramos para tratamento da esterilidade feminina e da impotência masculina e atribuindo-a propriedades tônicas, febrífugas e estomáticas (Lorenzi \& Matos, 2002).

O presente trabalho teve como objetivo revisar na literatura, em artigos de cunho científico, relatos sobre os aspectos morfológicos, atividade terapêutica e possíveis interações medicamentosas referente à planta Baccharis trimera, bem como, a rota biossintética de um dos metabólitos secundários, a hispidulina.

\section{Descrição botânica}

O gênero Baccharis, incluído na tribo Astereae da família Asteraceae, é constituído por cerca de 500 espécies. Uma das mais importantes é Baccharis trimera (Less.) DC., também denominada Baccharis genistelloides var. trimera (Less.) Baker, com grande utilização na medicina tradicional e na produção de fitoterápicos (Borella et al., 2006).

As espécies do gênero Baccharis têm porte arbustivo, com altura entre 0,5 e 4,0 metros. Arbusto bastante ramificado na base possui caules e ramos verdes com expansões trialadas. As inflorescências
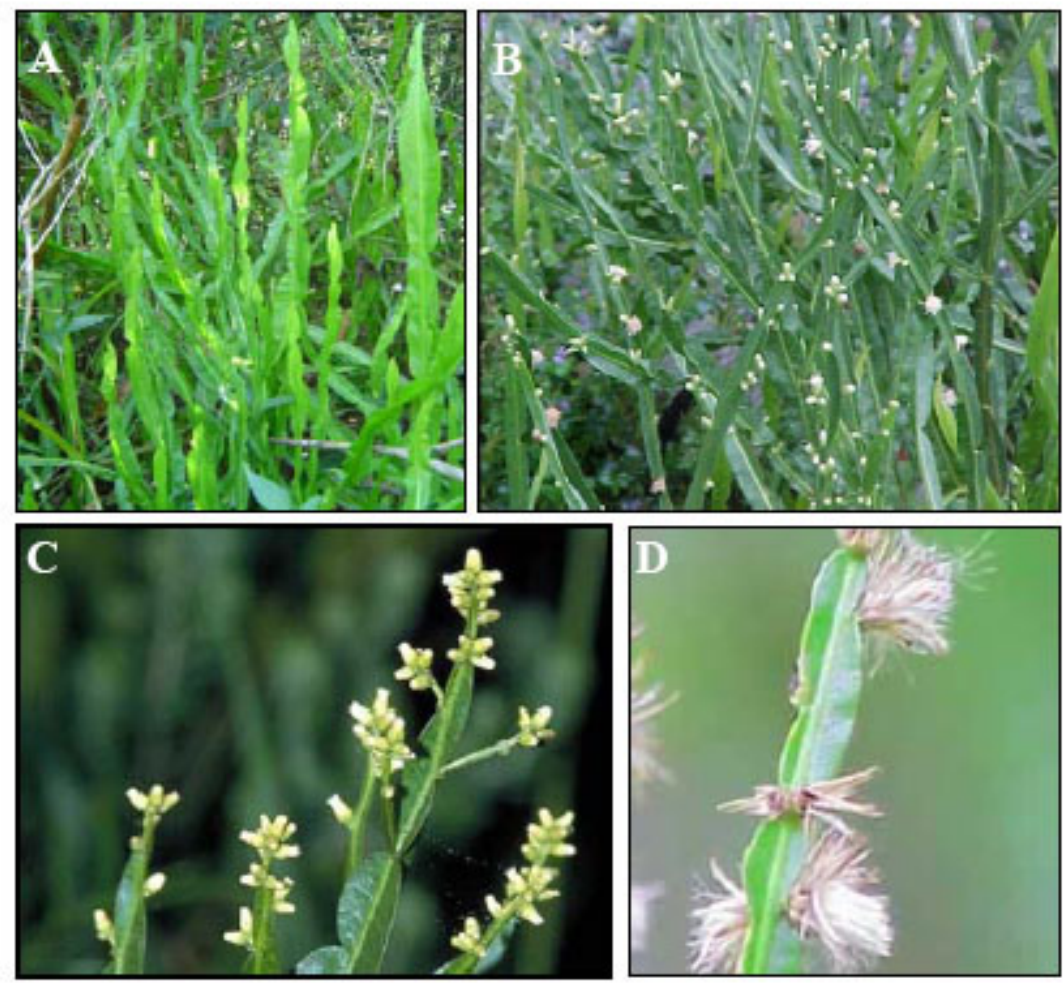

FIGURA 1. Plantas de Baccharis trimera em estádio vegetativo $(A)$ e reprodutivo (B), ramo de inflorescência (C) e detalhe dos frutos aquênios (D) (adaptado de Carreira, 2007). 
são do tipo capítulo, dispostas lateralmente nos ramos, de cor esbranquiçada (Figura 1) (Lorenzi \& Matos 2002). Apresenta ampla dispersão nos estados de Santa Catarina, Paraná, São Paulo e Rio Grande do Sul (Verdi et al., 2005).

\section{Componentes químicos (rota biossintética)}

A fitoquímica do gênero Baccharis tem sido extensivamente estudada desde o início do século vinte e, atualmente, mais de 150 compostos já foram isolados e identificados. Embora o gênero compreenda mais de 500 espécies, Verdi et al. (2005) relataram que apenas cerca de 120 espécies de Baccharis foram estudadas quimicamente. Os compostos mais frequentes são os flavonóides e os terpenóides, como monoterpenos, sesquiterpenos, diterpenos e triterpenos (Moreira et al., 2003; Verdi et al., 2005). Dentre essas espécies, cerca de 30 tiveram a atividade biológica estudada, destacando-se as atividades alelopática, analgésica, antidiabética, antifúngica, anti-inflamatória, antileucêmica, antimicrobiana, antimutagênica, antioxidante, antiviral, citotóxica, espasmolítica, gastroprotetora, hepatoprotetora, inseticida e vasorrelaxante (Kupchan et al., 1976; Soicke \& Leng-Peschlow, 1987; He et al., 1996; De las Heras et al., 1998; Nakasugi \& Komai, 1998; Torres et al., 2000; Weimann et al., 2002; Feresin et al., 2003; Oliveira et al., 2005).

A composição química da carqueja pode ser considerada regio-seletiva, ou seja, na parte aérea os constituintes químicos encontrados são predominantemente flavonóides (hispidulina, rutina, eupatorina, luteolina, nepetina, apigenina, kaempferol, cirsimaritina, cirsiliol, eriodictiol, 5-hidroxi-3', 4',6,7-tetrametoxiflavona, quercetina, 3-o-metilquercetina, genkwanina e 7,4'-di-o-metilapigenina), diterpenos (bacrispina, 1-desoxibacrispina, ácido hautriwaico e sua lactona), lactonas diterpênicas do tipo transclerodano (malonil clerodanos), estigmasterol, óleo essencial composto por á-pineno, â-pineno, canfeno, limoneno, acetato de carquejilo, carquejol, â-ocimeno, ledol e uma saponina derivada do ácido equinocístico. Por outro lado, no sistema radicular encontram-se diésteres terpênicos relacionados com o carquejol (Alonso \& Desmarchelier, 2006).

Contudo, os flavonóides estão entre os metabólitos secundários encontrados em maior quantidade na Baccharis trimera e, que apresentam maior atividade terapêutica. Podem ser divididos em várias classes, de acordo com o grau de oxidação e de insaturação do heterociclo (Figura 2). Sendo assim, ocorrem flavonóis (e.g. quercetina), flavonas (e.g. luteolina), isoflavonas (e.g. genisteína), flavanonas (e.g. naringenina), antocianidinas (e.g. cianidina), flavanóis (e.g. epicatequina) e as proantocianidinas, ou taninos condensados, que são flavanóis poliméricos (Marques, 2008).

A biossíntese dos flavonóides (Figura 3) ocorre por uma via mista, a via do ácido chiquímico e a do acetato. $O$ ácido chiquímico é o precursor do composto inicial da síntese dos flavonóides, a fenilalanina. Este aminoácido aromático depois de desaminado pela fenilalanina-amónia-líase (PAL) produz ácido cinâmico, que por ação da 4-hidroxilase cinamato é convertido em ácido p-cumárico. Estas duas enzimas estão associadas, o ácido cinâmico não é libertado pela PAL, mas sim transferido diretamente para o centro ativo da segunda enzima (Marques, 2008).

Posteriormente, ocorre a adição da CoA, catalisada pelo $p$-cumarato-CoA liase, originando a $p$-cumaroil-CoA, que ao reagir com três moléculas de malonil-CoA forma a naringenina chalcona. Esta reação é catalizada pela chalcona sintetase. Finalmente, ocorre a ciclização do anel C, catalizada pela chalcona isomerase, para formar a naringenina que por fim origina a hispidulina, por meio de metoxilação (Figura 3). Desta forma, nos flavanóides, o anel A é formado via acetato, enquanto o $B$ resulta da via chiquimato e os três átomos de carbono que ligam o anel $A$ ao $B$ derivam do fosfoenolpiruvato (Marques, 2008).

\section{Ações farmacológicas}

As diferentes propriedades atribuídas à carqueja na medicina tradicional vêm sendo estudadas e algumas foram validadas, como consequência dos resultados obtidos (Lorenzi \& Matos 2002). A validação do efeito hipoglicemiante foi feita com extratos de B. trimera (Xavier, 1967; Oliveira et al., 1995; Dickel et al., 2007). Utilizando extrato aquoso cru da planta em animais, Soicke \& Leng-Peschlow (1987) validaram suas propriedades hepatoprotetoras. As propriedades digestivas, antiúlcera e antiácida foram validadas em estudos com cobaias, ao mostrar que os extratos da planta reduziram a secreção gástrica e tiveram efeito analgésico (Gamberini et al., 1991) e anti-inflamatório (Gené et al., 1992). Extratos alcoólicos de carqueja apresentam potencial antimicrobiano, conforme comprovaram Bara \& Vanetti (1997) e, Avancini et al. (2000) confirmaram essa atividade in vitro a partir do decocto da planta.

De acordo com Alonso \& Desmarchelier (2006), algumas atividades farmacológicas da carqueja dentre as quais é possível destacar a atividade hepática, pois os flavonóides, em especial a hispidulina, possuem ação hepatoprotetora e antioxidante. Também foi evidenciada atividade antimicrobiana e inseticida, de modo que lactonas sesquiterpênicas apresentam atividade inibitória 


\begin{tabular}{llll}
\hline \multicolumn{4}{c}{ Flavonóis } \\
\hline & $\mathrm{R}_{1}$ & $\mathrm{R}_{2}$ & $\mathrm{R}_{3}$ \\
\hline Quercetina & $\mathrm{OH}$ & $\mathrm{OH}$ & $\mathrm{OH}$ \\
Kaempferol & $\mathrm{OH}$ & $\mathrm{H}$ & $\mathrm{OH}$ \\
\hline \multicolumn{5}{c}{ Flavonas } \\
\hline Luteolina & $\mathrm{H}$ & $\mathrm{OH}$ & $\mathrm{OH}$ \\
Apigenina & $\mathrm{H}$ & $\mathrm{H}$ & $\mathrm{OH}$ \\
\hline
\end{tabular}

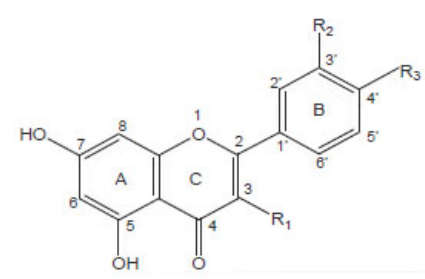

\begin{tabular}{ccc}
\hline \multicolumn{3}{c}{ Flavanóis } \\
\hline & $\mathrm{R}_{1}$ & $\mathrm{R}_{4}$ \\
\hline Catequina & $\mathrm{OH}$ & $\mathrm{H}$ \\
Epicatequina & $\mathrm{OH}$ & $\mathrm{H}$ \\
EGC & $\mathrm{OH}$ & $\mathrm{OH}$ \\
ECG & Galato & $\mathrm{H}$ \\
EGCG & Galato & $\mathrm{OH}$ \\
\hline
\end{tabular}

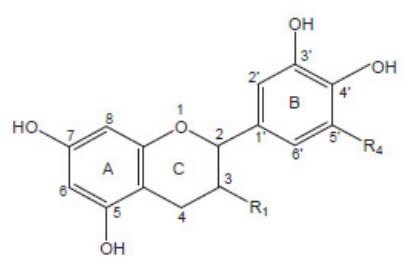

\section{Flavonóides}

\begin{tabular}{ccc}
\hline \multicolumn{3}{c}{ Antocianidinas } \\
\hline & $\mathrm{R}_{2}$ & $\mathrm{R}_{4}$ \\
\hline Cianidina & $\mathrm{OH}$ & $\mathrm{H}$ \\
Malvidina & $\mathrm{OCH}_{3}$ & $\mathrm{OCH}_{3}$ \\
Pelargonidina & $\mathrm{H}$ & $\mathrm{H}$ \\
\hline
\end{tabular}

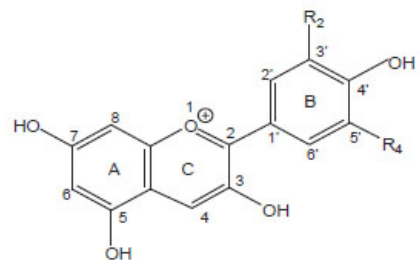

\begin{tabular}{ccc}
\hline & Isoflavonas & \\
\hline & $\mathrm{R}_{1}$ & $\mathrm{R}_{2}$ \\
\hline Daidzeina & $\mathrm{H}$ & $\mathrm{H}$ \\
Gliciteina & $\mathrm{H}$ & $\mathrm{OCH}_{3}$ \\
Genisteina & $\mathrm{OH}$ & $\mathrm{H}$ \\
\hline
\end{tabular}

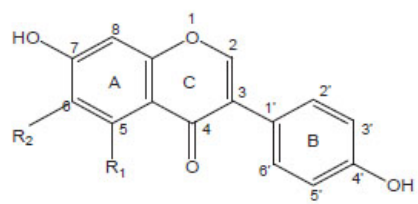

\begin{tabular}{cccc}
\hline \multicolumn{4}{c}{ Flavanonas } \\
\hline & $\mathrm{R}_{1}$ & $\mathrm{R}_{2}$ & $\mathrm{R}_{3}$ \\
\hline Taxifolina & $\mathrm{OH}$ & $\mathrm{OH}$ & $\mathrm{OH}$ \\
Naringenina & $\mathrm{H}$ & $\mathrm{H}$ & $\mathrm{OH}$ \\
\hline
\end{tabular}

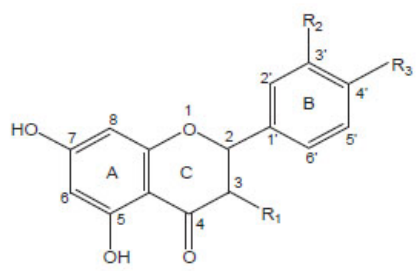

FIGURA 2. Diferentes classes de flavonóides (adaptado de Huber, 2008).

frente às cercárias de Schistosoma mansonii, e do crescimento do Trypanosoma cruzi (Alonso \& Desmarchelier, 2006).

Lactonas diterpênicas e flavonas, em especial eupatorina, demonstraram atividade molusquicida sobre Biomphalaria glabrata. Além disso, os diterpenóides exibiram atividade repelente frente às larvas de Tenebrio molitor. Em oncologia experimental verificou-se que hispidulina, gencavanina, cirsimaritina e apigenina apresentaram ação redutora da mutagenecidade demonstrada pela substância 3-amino-1-metil$5 \mathrm{H}$-pirido(4,3-b)-indol no teste de Salmonella typhimurium. Também foi relatada ação antihipertensiva, que através de atividade diurética resultou em hipotensão arterial. No sistema digestivo apresenta atividade antiulcerativa, através da infusão da planta inteira. Uma mescla de diterpenos obtida do extrato clorofórmico da parte aérea demonstrou atividade antiespasmódica, por bloquear as contrações induzidas pelo cálcio (Alonso \& Desmarchelier, 2006). 


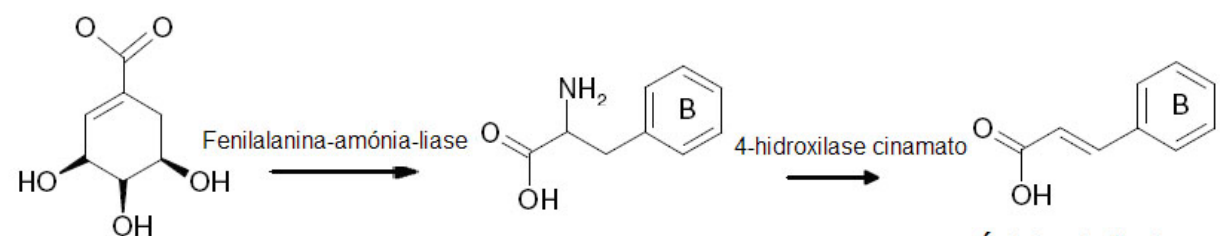
Chiquimato Fenilalanina

Ácido cinâmico

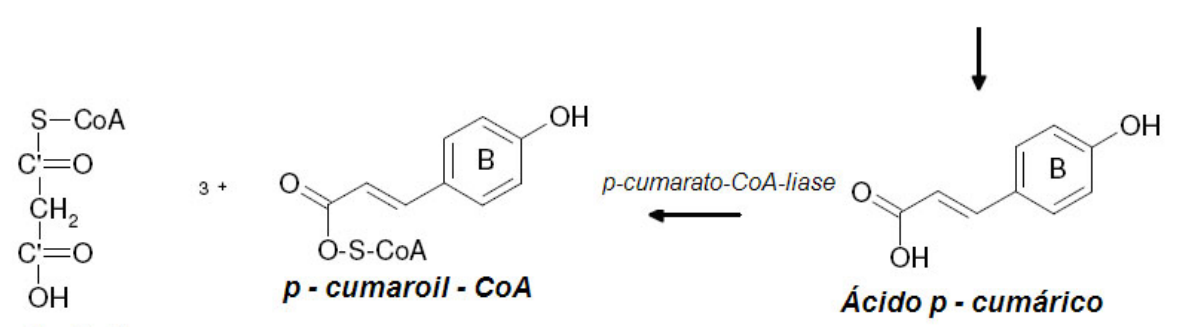

\section{Malonil - COA}

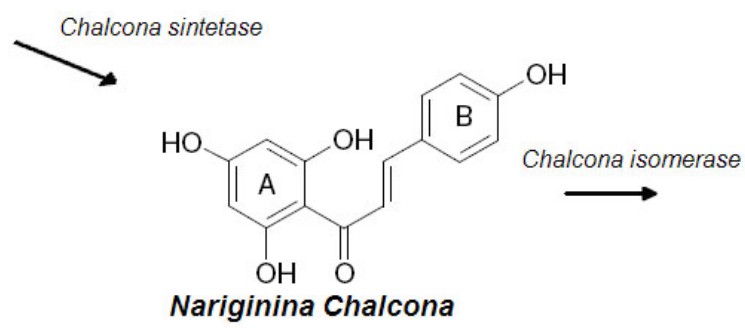<smiles>O=C1CC(c2ccc(O)cc2)Oc2cc(O)cc(O)c21</smiles>

Nariginina<smiles>O=c1cc(-c2ccc(O)cc2)oc2cc(O)c(O)c(O)c12</smiles>

Hispidulina

FIGURA 3. Biossíntese dos flavonóides (adaptado de Marques, 2008).

\section{Contraindicações e interações}

A carqueja possui baixa toxicidade por via oral, porém uma moderada toxicidade por via intraperitoneal foi observada. Esse efeito maléfico foi comprovado em estudo com a administração do extrato de carqueja em ratas, que teve efeito abortivo, sendo contraindicada durante a gestação e a lactação (Peron et al., 2008).

Em pacientes hipertensos que estejam em tratamento com anti-hipertensivos pode ocorrer sinergismo e elevar o efeito do fármaco, havendo a necessidade de ajuste das doses em caso de ingestão simultânea de extratos com carqueja (Alonso \& Desmarchelier, 2006).

$\mathrm{O}$ uso da carqueja concomitantemente a inibidores da síntese de proteína (tetraciclina, cloranfenicol e netilmicim) também acarreta uma interação agonista (Betoni, 2006).

\section{CONCLUSÃO}

A revisão bibliográfica possibilitou caracterizar morfologicamente a carqueja, expor algumas propriedades terapêuticas e, descrever a rota biossintética do flavonóide hispidulina, que se trata de um dos principais metabólitos secundários, responsável pelo efeito hepatoprotetor e antioxidante. A compreensão e conhecimento das enzimas e substratos envolvidos na biossíntese desse metabólito caracterizamse como importantes ferramentas no controle de qualidade e na padronização da terapêutica da carqueja. Apesar dos estudos desenvolvidos com a planta, é importante a condução de novas pesquisas buscando determinar potenciais ações terapêuticas da carqueja e formas de ação. Em geral, no Brasil, considerando a grande diversidade de espécies potenciais, há uma maior necessidade de estudos 
relacionados à composição química e atividade terapêutica de plantas medicinais.

\section{REFERÊNCIA}

ALONSO, J.; DESMARCHELIER, C. Plantas medicinales autóctonas de la Argentina. Bases científicas para su aplicación en atención primaria de la salud. Buenos Aires: LOLA, 2006. 663p.

AVANCINI, C.A.M.; WIEST, J.M.; MUNSTOCK, E. Atividade bacteriostática do decocto de Baccharis trimera (Less.) D.C., Compisitae, carqueja, como desinfetante ou antiséptico. Arquivo Brasileiro de Medicina Veterinária e Zootecnia, v.52, n.3, p.230-4, 2000.

BACCHI, E. M. Controle de qualidade de fitoterápicos. In: Di Stasi, L.C. (Org.). Plantas medicinais: arte e ciência, um guia de estudo interdisciplinar. São Paulo: Fundação Editora Unesp, 1996, 169-89p.

BARA, M.T.F., VANETTI, M.C.D. Estudo da atividade antibacteriana de plantas medicinais, aromáticas e corantes naturais. Revista Brasileira de Farmacognosia, v.7/8, n.1, p.22-34, 1998.

BETONI, J.E.C.; MANTOVANI, R.P.; BARBOSA, L.N.; STASI, L.C.D.; FERNANDES JUNIOR, A. Synergism between plant extract and antimicrobial drugs used on Staphylococcus aureus diseases. Memórias do Instituto Oswaldo Cruz, v.101, n.4, p.387-90, 2006.

BORELLA, J.C.; DONATA, P.D.; NOVARETTI, A.A.G.; MENEZES JUNIOR, A.; FRANÇA, S.C.; RUFALO, C.B.; SANTOS, P.A.S.; VENEZIANI, R.C.S.; LOPES, N.P. Variabilidade sazonal do teor de saponinas de Baccharis trimera (Less.) DC (Carqueja) e isolamento de flavona. Revista Brasileira de Farmacognosia, v.16, n.4, p.557-61, 2006.

CUNHA, A.P.; SILVA, R.A.; ROQUE, O.R. Plantas e Produtos Vegetais em Fitoterapia. 1. ed. Lisboa: Serviço de Educação e Bolsas, Fundação Calouste Gulbenkian, 2003, 9-11p.

CUNHA, A.P. Aspectos Históricos Sobre Plantas Medicinais, Seus Constituintes Activos E Fitoterapia. Disponível em: <http://www.esalq.usp.br/siesalq/pm/ aspectos_historicos.pdf>, 2005. Acesso em: 15 de setembro de 2010.

DE LAS HERAS, B.; SLOWING, K.; BENEDÍ, J.; CARRETERO, E.; ORTEGA, T.; TOLEDO, C.; BERMEJO, P.; IGLESIAS, I.; ABAD, M.J.; GÓMEZSERRANILLOS, P.; LISO, P.A.; VILLAR, A.; CHIRIBOGA, $X$. Antiinflammatory and antioxidant activity of plants used in traditional medicine in Ecuador. Journal of Ethnopharmacology, v. 61, n.2, p.161-6, 1998.

DICKEL, M.L.; RATES, S.M.K; RITTER, M.R. Plants popularly used for loosing weight purposes in Porto Alegre, South Brazil. Journal of Ethnopharmacology, v.109, n.1, p.60-71, 2007.

FERESIN, G.E.; TAPIA, A.; JIMÉNEZ, A.; RAVELO, A.G.; ZACCHINO, S.; SORTINO, M.; SCHMEDAHIRSCHMANN, G. Constituents of the Argentinian medicinal plant Baccharis grisebachii and their antimicrobial activity. Journal of Ethnopharmacology, v.89, n.1, p.73-80, 2003.

GAMBERINI, M.T. Inhibition of gastric secretion by a water extract from Baccharis triptera Mart. Memórias do Instituto Oswaldo Cruz, v.86, n.2, p.137-9, 1991. GENÉ, R.M.; MARIN,E.; ADZET, T. Anti-inflamatory effect of aqueous extracts of three species of the genus Baccharis. Planta Medica, v.58, n.6, p.565-6, 1992.

HE, K.; MONTENEGRO, G.; HOFFMMANN, J.J.; TIMMERMANN, B.N. Diterpenoids from Baccharis linearis. Phytochemistry, v.41, n.4, p.1123-7, 1996.

HUBER, L.S.; RODRIGUEZ-AMAYA, D.B. Flavonóis e flavonas: fontes brasileiras e fatores que influenciam a composição em alimentos. Alimentos e Nutrição, v.19, n.1, p.97-108, 2008.

KUPCHAN, S.M.; JARVIS, B.B.; DAILEY, R.G.; BRIGHT, J.W.; BRYAN, R.F; SHIZURI, Y. Baccharin, a novel potent antileukemic trichothecene triepoxide from Baccharis megagotarnica. Journal of American Chemical Society, v.98, n.22, p.7092-3, 1976.

LORENZI, H.; MATOS, F.J.A. Plantas medicinais do Brasil: nativas e exóticas. 1. ed. São Paulo: Instituto Plantarum, Nova Odessa, 2002, p.512.

MARQUES, O.C.P. Desenvolvimento de formas farmacêuticas sólidas orais de Uncaria tomentosa com atividade antioxidante. 2008. 210p. Dissertação (Mestrado - Área de Concentração em Farmácia) Departamento de Farmácia, Universidade de Coimbra, Coimbra, Portugal.

CARREIRA, R.C. Baccharis trimera (Less.) DC. (Asteraceae): estudo comparativo dos óleos voláteis, atividade biológica e crescimento de estacas de populações ocorrentes em áreas de Cerrado e Mata Atlântica. 2007, 199p. Tese (Doutorado - Área de Concentração em Botânica) - Departamento de Biodiversidade Vegetal e Meio Ambiente, Universidade de São Paulo, São Paulo, Brasil.

MOREIRA, F.P.M.; COUTINHO, V.; MONTANHER, A.B.P.; CARO, M.S.B.; BRIGHENTE, I.M.C.; PIZZOLATTI, M.G. Flavonóides e triterpenos de Baccharis pseudotenuifolia - Bioatividade sobre Artemisia salina. Química Nova, v.26, n.3, p.309-11, 2003.

NAKASUGI, T.; KOMAI, K. Antimutagens in the brazilian folk medicinal plant carqueja (Baccharis trimera Less.). Journal of Agriculture and Food Chemistry, v.46, n.7, p.2560-4, 1998.

OLIVEIRA, A.C.P.; ENDRINGER, D.C.; AMORIM, L.A.S.; BRANDÃO, M.G.L.; COELHO, M.M. Effect of the extracts and fractions of Baccharis trimera and Syzygium cumini on glycaemia of diabetic and non-diabetic mice. Journal of Ethnopharmacology, v.102, n.3, p.465-9, 2005.

VILEGAS, W.; CARDOSO, C.A.L. Controle químico de qualidade de fitoterápicos e plantas medicinais. In: Yunes, R.A.; Cechinel Filho, V.C. (orgs). Química de produtos naturais, novos fármacos e a moderna farmacognosia. 1. ed., Itajaí: Universidade do Vale do Itajaí, 2007, p. 157-9.

PERÓN, A.P.; FELIPES, J.; MATTGE, G. I.; CANTAGALLI. L.B.; MARIUCCI, R.G.; VICENTINI, V.E.P. Avaliação mutagênica das plantas medicinais Baccharis trimera Less. E Solanum melongena L., em células de medula óssea de ratos Wistar. Revista Brasileira de Biociências, v.6, n.2, p.127-30, 2008.

SOICKE H.; LENG-PESCHLOW E. Characterisation of flavonoids from Baccharis trimera and their antihepatotoxic properties. Planta Medica, v.53, n.1, p.37-9, 1987. 
TORRES, L.M.B.; GAMBERINI, M.T.; ROQUE, N.F.; LIMA-LANDMAN, M.T.; SOUCCAR, C.; LAPA, A.J. Diterpene from Baccharis trimera with a relaxant effect on rat vascular smooth muscle. Phytochemistry, v.55, n.6, p.617-9, 2000.

VERDI, L.G.; BRIGHENTE, I.M.C.; PIZZOLATTI, M.G. Gênero Baccharis (Asteraceae): aspectos químicos, econômicos e biológicos. Química Nova, v.28, n.1, p.85-94, 2005

XAVIER, A.A. PECKOLT OL, CANALI J. Effect of an extract of Baccharis genistelloides on the glucose level of the blood. Comptes Rendus des Séances de la Société de Biologie et de ses Filiales, v.161, n.4, p.972-4, 1967. 